\title{
Abnormal Cortical Processing of the Syllable Rate of Speech in Poor Readers
}

\author{
Daniel A. Abrams, ${ }^{1}$ Trent Nicol, ${ }^{1}$ Steven Zecker, ${ }^{1}$ and Nina Kraus ${ }^{1,2,3}$ \\ ${ }^{1}$ Auditory Neuroscience Laboratory, Department of Communication Sciences, and Departments of ${ }^{2}$ Neurobiology and Physiology and ${ }^{3}$ Otolaryngology, \\ Northwestern University, Evanston, Illinois 60208
}

Children with reading impairments have long been associated with impaired perception for rapidly presented acoustic stimuli and recently have shown deficits for slower features. It is not known whether impairments for low-frequency acoustic features negatively impact processing of speech in reading-impaired individuals. Here we provide neurophysiological evidence that poor readers have impaired representation of the speech envelope, the acoustical cue that provides syllable pattern information in speech. We measured cortical-evoked potentials in response to sentence stimuli and found that good readers indicated consistent right-hemisphere dominance in auditory cortex for all measures of speech envelope representation, including the precision, timing, and magnitude of cortical responses. Poor readers showed abnormal patterns of cerebral asymmetry for all measures of speech envelope representation. Moreover, cortical measures of speech envelope representation predicted up to $41 \%$ of the variability in standardized reading scores and $50 \%$ in measures of phonological processing across a wide range of abilities. Our findings strongly support a relationship between acoustic-level processing and higher-level language abilities, and are the first to link reading ability with cortical processing of low-frequency acoustic features in the speech signal. Our results also support the hypothesis that asymmetric routing between cerebral hemispheres represents an important mechanism for temporal encoding in the human auditory system, and the need for an expansion of the temporal processing hypothesis for reading disabilities to encompass impairments for a wider range of speech features than previously acknowledged.

\section{Introduction}

Reading proficiency relies on the confluence of rudimentary perceptual abilities and higher-order linguistic function (Schlaggar and McCandliss, 2007). While normal reading is thought to rely on an array of abilities, it is widely thought that reading-impaired individuals suffer from a specific deficit in representing or recalling the precise phonological structure of words (Ramus, 2001). This "phonological deficit" is thought to impair reading acquisition: if the mental representation of individual speech sounds is impaired, then the necessary step of learning how to match these sounds on to letters will also be impaired.

While it is widely accepted that impaired readers suffer from a phonological deficit, it is not known what causes this deficit. One influential hypothesis (Tallal et al., 1998) poses that abnormal perception of rapid acoustic events present in speech (on the order of tens of milliseconds) precludes normal development of phonological systems since many phonological contrasts rely on resolving acoustic events occurring on this time scale (Phillips and Farmer, 1990). Supporting this hypothesis, a body of evidence has accumulated that shows that many poor readers are impaired in the

Received Oct. 30, 2008; revised April 24, 2009; accepted April 25, 2009.

This work is supported by National Institutes of Health Grant R01 DC01510-10 and National Organization for Hearing Research Grant 340-B208. We thank the anonymous reviewers who provided helpful comments on previous drafts of this manuscript. We also thank the children who participated in this study and their families.

Correspondence should be addressed to Daniel A. Abrams at his present address: Stanford Cognitive and Systems Neuroscience Laboratory, 780 Welch Road, Room 201, Palo Alto, CA 94304. E-mail: daa@stanford.edu.

D01:10.1523/JNEUROSCI.5242-08.2009

Copyright $\odot 2009$ Society for Neuroscience $\quad$ 0270-6474/09/297686-08\$15.00/0 perception of rapid acoustic events in speech and nonspeech signals (Tallal and Piercy, 1973; Tallal, 1980; Kraus et al., 1996).

A second auditory-based hypothesis states that abnormal perception of low-frequency temporal features in speech and nonspeech signals (on the order of hundreds of milliseconds) additionally contributes to reading impairments (Goswami, 2002). In speech, these acoustic features, known as the speech envelope, provide syllable pattern information and are essential for normal perception (Drullman et al., 1994). Supporting this hypothesis, it has been shown that perceptual deficits for low-frequency temporal cues in nonspeech acoustic signals account for significant variance in reading scores (Goswami et al., 2002; Witton et al., 2002; Richardson et al., 2004; Corriveau et al., 2007). It is not known whether this impairment directly impacts speech processing.

Recent work has provided insight into neural mechanisms governing temporal processing of speech in the unimpaired human auditory system. A mechanism for processing temporal information is the asymmetric routing of this information between cerebral hemispheres (Poeppel, 2003). Rapid features in speech are lateralized to left-hemisphere auditory areas (Belin et al., 1998; Liégeois-Chauvel et al., 1999; Joanisse and Gati, 2003; Abrams et al., 2006), while the speech envelope is lateralized to the right hemisphere (Abrams et al., 2008). This model of temporal processing affords an opportunity to test competing theories of reading impairment. The rapid-processing hypothesis, as well as theories of reading impairment that do not subscribe to auditory-based phonological deficits (White et al., 2006), predict "normal" representation of the speech envelope in poor readers. Alternatively, proponents of syllable-level deficits (Goswami et 
al., 2002) predict that poor readers demonstrate impairment for the neural representation of the speech envelope. To test these hypotheses, we measured cortical potentials that represent the speech envelope in a group of children spanning a wide range of reading abilities and examined the relationship between reading and phonological abilities and measures of envelope processing in cortex.

\section{Materials and Methods}

The research protocol was approved by the Institutional Review Board of Northwestern University. Parental consent and the child's assent were obtained for all evaluation procedures, and children were paid for their participation in the study.

\section{Participants}

Participants consisted of 23 children between 9 and 15 years old who reported no history of neurological or otological disease and were of normal intelligence [scores $>85$ on either the Test of Nonverbal Intelligence (Brown et al., 1997) or Brief Cognitive Scale (Woodcock and Johnson, 1977)]. Since cerebral dominance for language is often reversed in left-handed and ambidextrous individuals, and many of the analyses performed here relate to cerebral asymmetries, all subjects completed a questionnaire to assess handedness.

Children were recruited from a database compiled in an ongoing project entitled Listening, Learning, and the Brain. Children who had previously participated in this project and had indicated interest in participating in additional studies were contacted via telephone. Children were selected for this study to provide a cohort with a wide range of reading abilities (supplemental Table 1, available at www.jneurosci.org as supplemental material). Neurophysiologic responses were collected in a single session, and measures of reading and phonological processing were measured in a subsequent session.

\section{Stimuli}

Stimuli consisted of the sentence stimulus "The young boy left home" produced in three modes of speech: conversational, clear, and compressed modes (supplemental Fig. 1, available at www.jneurosci.org as supplemental material). These three modes of speech have different speech envelope cues and were used as a means to elicit a variety of cortical activation patterns. Briefly, clear speech has greater temporal envelope modulations than conversational speech, and compressed speech, which approximates rapidly produced speech, is characterized by more rapid speech envelope cues than conversational speech. A full account of the acoustical characteristics and production of these sentences has been provided previously (Abrams et al., 2008).

\section{Neurophysiological recording and data-processing procedures}

All of the recording and data-processing techniques used to describe cortical representation of the speech envelope are identical to those described in detail (Abrams et al., 2008). Briefly, sentence stimuli were presented unilaterally to the right ear through ER-2 insert earphones (Etymotic Research) at $80 \mathrm{~dB}$ sound pressure level (SPL). Presentation of the three speech modes was pseudorandomly interleaved. An interval of $1 \mathrm{~s}$ separated the presentation of each sentence. Subjects were tested in a sound-treated booth and were instructed to ignore the sentences. To promote subject stillness during long recording sessions as well as diminish attention to the auditory stimuli, subjects watched a videotape movie of his or her choice and listened to the soundtrack to the movie in the nontest ear with the sound level set at $<40 \mathrm{~dB}$ SPL. This paradigm for measuring cortical evoked potentials has been used in previous studies investigating cortical asymmetry for speech sounds (Bellis et al., 2000; Abrams et al., 2006; Abrams et al., 2008) as well as other forms of cortical speech processing (Kraus et al., 1996; Banai et al., 2005; Wible et al., 2005). Cortical responses to speech stimuli were recorded with 31 tin electrodes affixed to an Electrocap (Electrocap International) brand cap (impedance $<5 \mathrm{k} \Omega$ ). Additional electrodes were placed on the earlobes and superior and outer canthus of the left eye. These act as the reference and eye blink monitor, respectively. Responses were collected at a sampling rate of $500 \mathrm{~Hz}$ for a total of 1000 repetitions each for clear, conver-
Table 1. Good and poor readers' subject characteristics

\begin{tabular}{lccr}
\hline & Good readers & Poor readers & $p$ value \\
\hline$n$ & 8 & 8 & \\
Age & $12.0(1.5)$ & $12.8(2.0)$ & $>0.35$ \\
Nonverbal IQ & $123.9(19.5)$ & $103.9(19.5)$ & 0.059 \\
Reading & $115.6(5.4)$ & $89.4(6.0)$ & $<0.001$ \\
Nonword reading & $125.1(9.1)$ & $91.0(3.9)$ & $<0.001$ \\
Phonological processing & $102.2(7.4)$ & $85.5(9.1)$ & $<0.002$ \\
\hline
\end{tabular}

sational, and compressed sentences. Neurophysiological responses were low-pass filtered at $40 \mathrm{~Hz}$ to isolate cortical contributions.

\section{Data analysis: measures of cortical speech envelope processing in} good and poor readers

The first goal of this study was to use established measures of speech envelope representation in the cortex (Abrams et al., 2008) to examine whether the speech envelope is abnormally represented in impaired readers, an hypothesis that is suggested in the literature (Goswami et al., 2002). We defined "good readers" as subjects in the top third of the larger group ( $n=23$ total; $n=8$ in each group) based on the average of single-word (Wilkinson, 1993) and nonword (Woodcock and Johnson, 1989) reading scores; we defined "poor readers" as subjects in bottom third based on these measures (see Table 1 for descriptive statistics of the subgroups). The following paragraphs describe the measures used to compare between good and poor reading groups.

All data analyses were performed using software written in Matlab (The MathWorks). Broadband stimulus envelopes were determined by performing a Hilbert transform on the broadband sentence waveforms (Drullman et al., 1994). The resulting amplitude envelopes were low-pass filtered at $40 \mathrm{~Hz}$ to isolate the speech envelope (Rosen, 1992) and to match the frequency characteristics of the processed responses. We calculated the frequency of maximal power, known as the modal frequency (Ahissar et al., 2001), of the envelope of each speech sentence stimulus by performing fast Fourier transforms (FFTs) of the low-pass filtered Hilbert envelope. FFTs were calculated using windows of $1 \mathrm{~s}$ and overlaps of $0.5 \mathrm{~s}$, consistent with a previous report (Ahissar et al., 2001).

A previous study showed strong right-hemisphere asymmetry for coding the speech envelope in unimpaired children (Abrams et al., 2008), and the current analyses were also designed to examine hemispheric effects for speech envelope representation. Therefore, data are presented for three temporal electrode pairs: (1) T3 and T4, (2) T5 and T6, and (3) $\mathrm{Tp} 7$ and Tp8 according to the modified International 10-20 recording system (Jasper, 1958). The modification is the addition of the Tp7-Tp8 electrode pair, in which Tp7 is located midway between T3 and T5, and Tp8 is located midway between T4 and T6. Two types of analyses were performed on the data: cross-correlation and RMS analyses, resulting in three measures of cortical speech-envelope representation. These measures were established in a previous study (Abrams et al., 2008). First, cross-correlations between the broadband speech envelope and cortical responses at each temporal electrode for the "envelope-following period" (250-1500 ms for conversational and clear stimuli, 250-750 ms for the compressed stimulus) were performed using the "xcov" function in Matlab. The peak in the cross-correlation function was found at each electrode between 50 and $150 \mathrm{~ms}$ lags, resulting in the first two measures. (1) Phase-locking precision was defined as the peak $r$ value, and (2) phaselocking timing was defined as the lag at the peak $r$ value. $r$ values were Fisher transformed before statistical analysis. Finally, (3) phase-locking magnitude, via RMS amplitude computation, at each electrode was calculated for two different time ranges: the "onset" period was defined by the time ranges $0-250 \mathrm{~ms}$ for all stimuli; and the "envelope-following" period was defined as $250-1500 \mathrm{~ms}$ for conversational and clear stimuli and 250-750 $\mathrm{ms}$ for the compressed stimulus.

Data analysis: relationship between measures of cortical speech envelope processing and reading and phonological processing

The first analysis was restricted to good and poor readers $(n=8$ in each group). It is conceivable that any between-group differences observed in 
this initial analysis could have been driven by a small number of total cases, and that there is in fact substantial overlap between the good and poor reader distributions. To examine the generality of the speech envelope-reading relationship, and to rule out the possibility that the group differences were driven by a few deviant cases, we examined the relationship between cortical measures and reading ability across all subjects $(n=23)$. To this end, we correlated individual subjects' values for the neurophysiological measures of speech envelope representation described above with standardized measures of reading and phonological processing. The following is a description of these cortical measures, each of which was subsequently Pearson's correlated with the standardized behavioral measures.

Phase-locking precision. Poor readers were most clearly differentiated from good readers based on the symmetry of their $r$ values in the compressed speech condition. Therefore, we first calculated mean left-hemisphere $r$ values (i.e., the mean of $\mathrm{T} 3, \mathrm{~T} 5$, and $\mathrm{Tp} 7$ ) and righthemisphere $r$ values (i.e., the mean of T4, T6, and Tp8) from the cross-correlation analysis of the compressed speech condition, and entered these values into the asymmetry index $(R-$ $L) /(R+L)$.

Phase-locking timing. Poor readers were differentiated from control subjects based on later right-hemisphere cortical lags and earlier left-hemisphere lags evident across all speech conditions. Therefore, we first calculated mean lefthemisphere lags (i.e., the mean of $\mathrm{T} 3, \mathrm{~T} 5$, and Tp7) and right-hemisphere lags (i.e., the mean of $\mathrm{T} 4, \mathrm{~T} 6$, and $\mathrm{Tp} 8$ ) from the cross-correlation analysis for each subject and each speech condition, and entered these values into the asymmetry index $(R-L) /(R+L)$.

Phase-locking magnitude. Poor readers were most clearly differentiated from good readers based on the symmetry of their envelope-following RMS amplitudes in the compressed speech condition in the two rostralmost electrode pairs. Therefore, we first calculated mean left-hemisphere amplitudes (i.e., the mean of $\mathrm{T} 3$ and $\mathrm{Tp} 7$ ) and right-hemisphere amplitudes (i.e., the mean of $\mathrm{T} 4$ and $\mathrm{Tp} 8$ ) to the compressed speech condition, and entered these values into the asymmetry index $(R-L) /(R+L)$.

\section{Statistical analysis}

The statistical design used a series of mixed-model and repeatedmeasures ANOVAs to assess group differences for cross-correlation and RMS measures between good and poor readers. When appropriate, post hoc ANOVAs were conducted using repeated measures whenever possible to maximize statistical power. Repeated-measures ANOVA $p$ values $<0.05$ were considered statistically significant.

Pearson's correlations were performed between measures of cortical speech envelope processing and standardized measures of reading and phonological processing. To prevent spurious results from correlation analyses and $t$ tests, which can be overly biased by outlying data points, all raw cortical asymmetry values beyond 2 SDs of the mean were moved to the $2 \mathrm{SD}$ point for that particular measure. Across cortical measures, two data points (out of a total of 69) were moved to the 2 SD point. Pearson's correlations $p$ values $\leq 0.05$ were considered statistically significant.

\section{Results}

The goal of this work was to examine the relationship between reading ability and obligatory cortical representation of slow temporal cues in speech. Toward this end, we analyzed the data in two ways. First, we reasoned that if such a relationship exists, then neurophysiologic differences would be evident when comparing subjects at the tails of the reading distribution. Therefore, we compared cortical responses between subjects in the top third ("good readers") and bottom third ("poor readers") of the larger group ( $n=23$ total; $n=8$ in good and poor reader groups; see Table 1 for descriptive statistics of the subgroups) based on the average of single-word (Wilkinson, 1993) and nonword (Woodcock and Johnson, 1989) reading scores. Second, we reasoned that if cortical coding of the speech envelope represents an important factor for the development of normal reading and phonology, it should not only differ between good and poor readers, but variance in measures of cortical envelope representation should be able to predict scores on standardized measures of reading and phonological processing across all subjects (i.e., across a wide range of behavioral abilities).

\section{Cortical envelope representation in "good" and "poor" readers}

Figure 1 shows grand average cortical responses to clear (top left) and compressed (top right) speech sentences from good and poor reading groups. These two conditions were plotted because they represent the most intelligible and most challenging speech conditions, and impaired populations often reveal abnormal perception and representation of speech only in difficult listening conditions (Watson et al., 1990; Cunningham et al., 2001; Bradlow et al., 2003).

\section{Speech envelope phase-locking precision}

Our previous work in unimpaired readers described three metrics of speech envelope representation in cortex (Abrams et al., 2008): (1) precision, (2) timing, and (3) magnitude. To investigate precision and timing of envelope phase locking, we crosscorrelated the broadband stimulus envelope with left- and righthemisphere temporal electrode responses, and plotted mean correlograms for clear (Fig. 1, bottom left) and compressed (Fig. 1 , bottom right) conditions in good and poor readers. For the clear speech condition, correlograms for both reading groups 

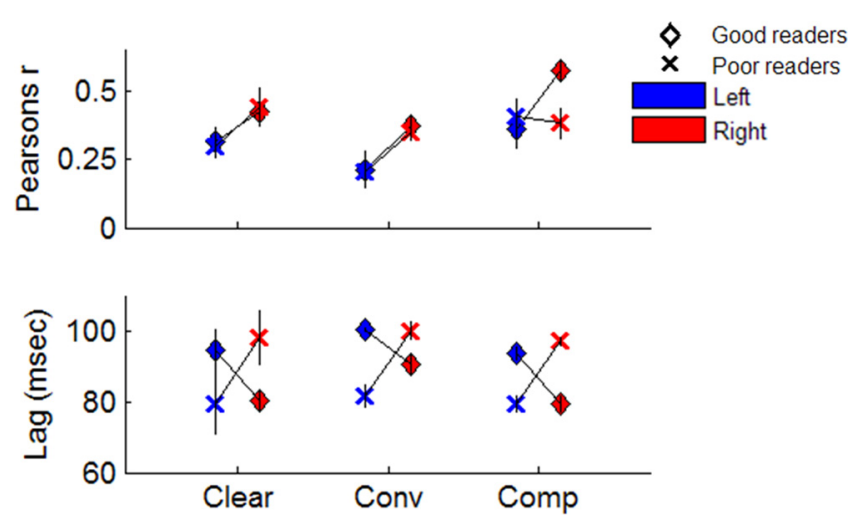

Figure 2. Average phase-locking precision (top) and timing (bottom) values. Values represent the average collapsed left- (blue; T3, T5, Tp7) and right- (red; T4, T6, Tp8) hemisphere results. Error bars represent 1 SEM.

indicated asymmetry for phase-locking precision, seen as the $y$-axis of the correlogram: correlogram peaks were considerably greater in right-hemisphere electrodes for both groups. These observations were investigated with a $2 \times(3 \times 2)$ mixed-model ANOVA [group $\times$ (electrode $\times$ hemisphere)] with repeated measures (RM) on the final two factors. The dependent variable in this analysis was the Fisher's $Z^{\prime}$ value associated with the peak Pearson's $r$ value for each subject from three paired electrode sites in response to the clear speech condition. Results confirmed our observations that Pearson's $r$ values were greater in the right hemisphere in the clear speech condition (main effect of hemisphere: $\left.F_{(1,14)}=9.83, p=0.007\right)$ (Fig. 2) and were similar between groups (main effect of group: $F_{(1,14)}=0.0001, p=0.992$; hemisphere $\times$ group interaction: $\left.F_{(1,14)}=0.27, p=0.61\right)$. The same rightward asymmetric pattern was seen for the conversational condition in both groups (main effect of hemisphere: $F_{(1,14)}=7.54, p=0.016$; main effect of group: $F_{(1,14)}=0.20, p=$ 0.66 ; hemisphere $\times$ group interaction: $\left.F_{(1,14)}=0.02, p=0.88\right)$.

In contrast, in the compressed speech condition, while good readers showed the same pattern of right-dominant asymmetry for precision of phase locking, poor readers showed a strikingly different pattern of activation. Specifically, phase-locking precision appeared to be represented symmetrically across cerebral hemispheres (Fig. 1, bottom right). These observations were confirmed with statistical analyses: in compressed speech, ANOVA results failed to show a main effect of hemisphere $\left(F_{(1,14)}=2.79\right.$, $p=0.12$ ) but did show a near-significant interaction (hemisphere $\times$ group: $\left.F_{(1,14)}=4.387, p=0.055\right)$. Post hoc analyses confirmed that good readers continued to show right-dominant asymmetry for precision of phase locking in the compressed condition $[3 \times 2$ RMANOVA (electrode $\times$ hemisphere) main effect of hemisphere: $\left.F_{(1,7)}=5.577, p=0.050\right]$. In contrast, $r$ values in poor readers were statistically similar between right- and lefthemisphere electrodes (main effect of hemisphere: $F_{(1,7)}=0.12$, $p=0.74)$. These data indicate that in clear and conversational speech conditions, right-hemisphere auditory cortex was dominant for phase-locking precision in both good and poor readers, but when presented with compressed speech, only good readers showed right-hemisphere asymmetry.

\section{Speech envelope phase-locking timing}

We investigated the timing of cortical phase locking to the speech envelope by analyzing the lags of subjects' correlogram peaks. A pattern that was evident across conditions was that good readers had earlier lags in right-hemisphere electrodes (Fig. 1, bottom) than left-hemisphere electrodes. In contrast, poor readers showed later lags in right-hemisphere electrodes than in the left (Fig. 2, bottom right). We performed a $2 \times(3 \times 3 \times 2)$ mixedmodel ANOVA (group $\times$ stimulus $\times$ electrode $\times$ hemisphere) with repeated measures on the final three factors with lags as the dependent variable and found a significant interaction of hemisphere $\times$ group $\left(F_{(1,14)}=16.44, p=0.001\right)$. Since there was no main effect of stimulus condition $\left(F_{(2,28)}=1.33, p=0.28\right)$, hemisphere $\times$ stimulus $\times$ group interaction $\left(F_{(2,28)}=0.04, p=0.96\right)$, or two-way interaction involving stimulus $(F<1.7, p>0.15$ for all two-way interactions), we collapsed results across the three stimulus conditions for post hoc analyses to improve statistical power. Consistent with our observations, poor readers' cortical responses were both later in the right hemisphere [post hoc $2 \times 3$ mixed-model ANOVA (group vs right-hemi electrodes): main effect of group: $F_{(1,46)}=6.675, p=0.013$ ] and earlier in the left hemisphere (main effect of group: $F_{(1,46)}=6.147, p=0.017$ ) than in good readers. This result indicates a fundamentally different pattern of timing for cortical speech envelope representation in poor readers than in good readers.

\section{Onset and speech envelope magnitude}

To examine the magnitude of activation across left- and righthemisphere auditory cortex, we performed RMS amplitude analyses on the "onset" and "envelope-following" portions of the response (Abrams et al., 2008) (Fig. 3). Mean RMS amplitude in the onset segment did not appear to indicate group differences (supplemental Fig. 2, available at www.jneurosci.org as supplemental material $)$, a result that was confirmed with a $2 \times(3 \times 3 \times$ 2) mixed-model ANOVA [group $\times$ (stimulus $\times$ electrode $\times$ hemisphere)] with repeated measures on the final three factors using onset RMS amplitude as the dependent variable $(F \leq 2.7$, $p>0.080$ for all main effects and interactions involving group). For the envelope-following period, we first observed that the two rostralmost electrode pairs, T3-T4 and Tp7-Tp8, appeared to show different patterns of asymmetry in the two reading groups across stimulus conditions. Consistent with this observation, an investigation with a $2 \times(3 \times 3 \times 2)$ mixed-model ANOVA using envelope-following RMS amplitude as the dependent variable showed a significant interaction of group $\times$ electrode $\times$ hemisphere $\left(F_{(2,28)}=4.28, p=0.024\right)$. Post hoc ANOVAs revealed that good readers had larger responses in right-hemisphere electrodes in two of three electrode pairs (both T3-T4 and Tp7-Tp8 electrode pairs: $F_{(1,7)} \geq 15.75, p \leq 0.005$; T5-T6: $F_{(1,7)}=1.99, p=$ $0.20)$, while poor readers were symmetric in all three electrode pairs $\left(F_{(1,7)} \leq 0.60, p \geq 0.46\right)$.

Additionally, it appeared that there were qualitatively different hemispheric response patterns in the compressed speech condition between good and poor readers. Specifically, it appeared that poor readers showed diminished asymmetry in the compressed condition, while good readers showed robust rightdominant asymmetry.

To examine this, we performed a post hoc $2 \times(2 \times 2)$ ANOVA [group $\times$ (electrode $\times$ hemisphere $)$ ] in which we isolated the magnitude analysis to the compressed speech condition and the two electrode pairs that indicated significant asymmetry in good readers ( $\mathrm{T} 3$ and $\mathrm{T} 4$; $\mathrm{Tp} 7$ and $\mathrm{Tp} 8$ ). Consistent with our observations, results indicated different patterns of asymmetry between good and poor readers in the compressed speech condition (hemisphere $\times$ group interaction: $F_{(1,14)}=5.94, p=0.029$ ). Within-group ANOVAs revealed that good readers had larger responses in right-hemisphere electrodes in the compressed 
speech condition $\left(F_{(1,7)}=12.40, p=\right.$ 0.01 ), while poor readers' response amplitudes were symmetric $\left(F_{(1,7)}=0.236, p=\right.$ $0.64)$.

Cortical envelope representation and its relationship to measures of reading and phonological processing

To examine the generality of the speech envelope-reading relationship, and to rule out the possibility that good versus poor reader group differences were driven by a few deviant cases, we examined the relationship between cortical measures and reading ability across all subjects $(n=23)$. Results indicate that all three measures of cortical function significantly correlated with this measure of reading (Fig. 4, top), and these measures of cortical function accounted for $17-41 \%$ of the variability in reading scores. Significant relationships were also found between two of the cortical measures, phase-locking precision and magnitude, and a standardized measure of phonological processing (Woodcock and Johnson, 1989) (composite score derived from the Incomplete Words and Sound Blending subtests; $r>0.5, p<0.01$ for cortical-phonological processing correlations) (Fig. 4, bottom). Phase-locking precision and magnitude asymmetries accounted for $29 \%$ and $50 \%$ of the variability in phonological processing scores, respectively.

\section{Effects of handedness}

Left-handed and ambidextrous individuals often have reversed (i.e., righthemisphere) cerebral dominance for language. It is not known how dominance for language is related to cerebral asymmetries for temporal information in acoustic signals, and we wanted to rule out handedness as a contributing factor to group differences described here. To this end, we reperformed all correlation analyses after removing the four subjects who reported as either left handed or ambidextrous. Re-

sults were identical to those described in the original analysis (which included all subjects) with one exception: the correlation between reading and phase-locking magnitude asymmetry failed to reach statistical significance $(p=0.170)$. Nevertheless, this particular cortical measure still predicted $>50 \%$ of the variance in phonological processing scores across right-handed subjects $(r=0.719, p<0.001)$.

\section{Effects of intelligence quotient}

It is noted that there was a marginal group difference with respect to nonverbal intelligence quotient (IQ) in good versus poor reader comparison ( $n=8$ in each group) (Table 1$)$, and the entire sample $(n=23)$ showed a significant correlation between reading scores and IQ $(r=0.418, p=0.047)$. Previous evidence suggests that reading impairments are associated with slightly lower IQ than normal subjects (Wechsler, 1991) such that our electrodes.
Poor readers



Figure 3. Average phase-locking magnitude for the envelope-following period measured at six electrode locations. The envelope-following period was defined as 250-1500 ms (clear and conversational speech conditions) or 250-750 ms (com-
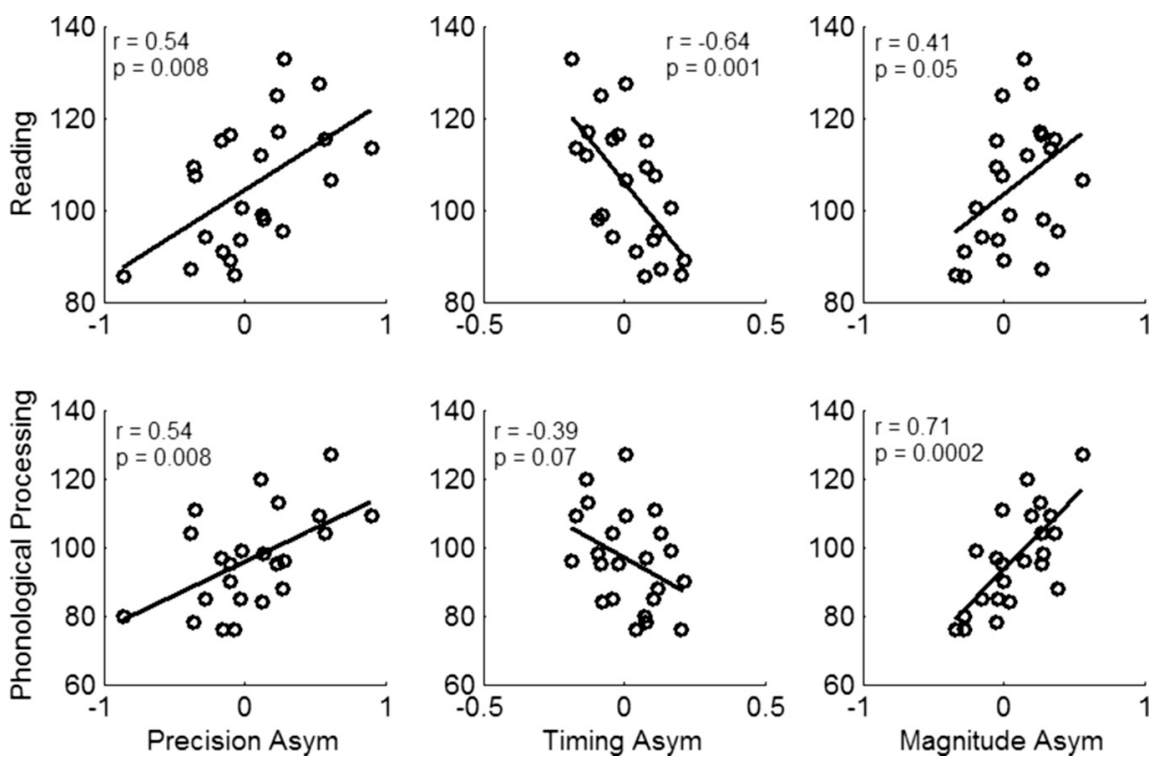

Figure 4. Three measures of cortical speech envelope representation and standardized measures of reading (top row) and phonological processing (bottom row). The ordinate for all plots is standard score. Left column, The abscissa is the left- and right-hemisphere $r$ values from the cross-correlation analysis of the compressed speech condition, entered into the asymmetry is the left- (T3 and Tp7) and right- (T4 and Tp8) hemisphere envelope-following RMS amplitude for the compressed speech condition, entered into the asymmetry index $(R-L) /(R+L)$.

sample is representative of this population. It is noted that the current developmental literature favors a definition of reading difficulty that is not IQ dependent (Gustafson and Samuelsson, 1999; Vellutino et al., 2000). Nevertheless, we wanted to rule out the possibility that IQ was driving correlations between cortical representations of the speech envelope and standardized measures of reading and phonological processing. First, we reperformed all correlation analyses while covarying for nonverbal IQ. Correlations results were the same after covarying for IQ with one exception: the correlation between reading and magnitude asymmetry failed to reach significance ( $p=0.127)$, although this cortical measure still predicted $>48 \%$ of the variance in phonological processing scores $(r=0.696, p<0.001)$. Second, we performed Pearson's correlations between IQ and the three cortical measures of speech envelope processing, and results showed no significant relationships between these variables $(p>0.10$ for 
all three cortical measures). Finally, we performed a linear multiple regression analysis in which reading score was the dependent variable and the three cortical measures and nonverbal IQ were predictors. Results indicated that together these predictors explained $50 \%$ of the variance in reading scores $\left(r=0.71 ; F_{(4,18)}\right.$ $=4.58, p=0.010)$. Furthermore, cortical lag contributed significantly to reading scores $(t=2.32, p=0.032)$, while the other variables, including nonverbal IQ, did not $(t<1.1, p>0.25$ for precision, magnitude, and IQ). Similar multiple regression results were seen when phonological processing score served as the dependent variable $\left(r=0.72, F_{(4,18)}=4.74, p<0.01\right.$; speech envelope magnitude: $t=2.8, p=0.013$; all other predictors, including nonverbal IQ: $t<0.5, p>0.6)$.

\section{Discussion}

We measured cortical evoked potentials that represent the speech envelope in a group of children with a range of reading abilities. Across speech conditions, good readers showed consistent, rightward asymmetry in auditory cortex for three measures of speech envelope representation. Poor readers showed a variety of response patterns that differed from good readers, including a measure that differed in all stimulus conditions (timing) and measures that were only abnormal in an acoustically challenging condition, compressed speech (precision and magnitude). Moreover, cortical speech envelope representation predicted up to $41 \%$ of the variability in standardized reading scores across subjects.

\section{The "asymmetric sampling in time" hypothesis and reading ability}

Current results add to an emerging body of literature describing neural mechanisms for processing temporal features in the human auditory system. This processing scheme is described in the "asymmetric sampling in time" (AST) hypothesis and states that low-frequency temporal features $(3-5 \mathrm{~Hz})$ in acoustical signals lateralize to right-hemisphere auditory areas, while faster features $(20-50 \mathrm{~Hz})$ lateralize to the left (Poeppel, 2003). These rates are critical for speech perception: slow rates provide essential syllable pattern information; fast temporal modulations characterize many phonemic contrasts (e.g., formant transitions, voice onset time).

Results are the first to test the AST hypothesis in an impaired population and show that cortical processing specified by this hypothesis predicts up to $41 \%$ of the variability in reading scores across reading abilities. The relationship between temporal acoustic processing and high-level cognitive abilities like reading is largely unknown. Historically a "bottom-up" relationship has been assumed in which precise temporal processing of acoustic features in speech sharpens phonological representations necessary for reading acquisition (Tallal et al., 1993). More recently, studies have shown that "top-down" mechanisms support the refining of acoustic feature representation (Polley et al., 2006; Luo et al., 2008; Nahum et al., 2008; Song et al., 2008; Tzounopoulos and Kraus, 2009), and that top-down signaling can contribute to subcortical acoustical representations important for reading and phonological processing (Cunningham et al., 2001; Banai et al., 2005, 2009; Banai and Kraus, 2006). While the current results cannot address where the sharpening of acousticlinguistic representations is initiated in the brain, they suggest a close relationship between low-level acoustical processing and high-level linguistic abilities.

\section{Reading ability and the speech envelope}

A major finding of this study was that different patterns of cortical activation were seen in good and poor readers. In the case of cortical response timing, poor readers' delays were seen across all speech conditions: timing of right-hemisphere cortical responses, which are presumed to be dominant for the processing of the speech envelope (Abrams et al., 2008), was later in poor readers than in good readers. While it is tempting to suggest that poor readers are delayed for speech envelope representation, this is complicated by the fact that left-hemisphere representations were earlier in poor than in good readers. It is also tempting to suggest that abnormal timing patterns in poor readers reflects reversed asymmetry for speech envelope encoding; however, this is confounded by the fact that other measures (e.g., precision and magnitude of envelope representation) were right-dominant and similar to good readers' responses in clear and conversational conditions. A simple explanation of speech envelope timing results is elusive given our current knowledge of temporal processing mechanisms and the biological foundations of reading impairments.

With respect to the precision and magnitude of speech envelope representation, results showed that poor readers had abnormal cortical responses only in compressed speech. In clear and conversational speech, good and poor readers showed similar patterns of envelope representation. This finding is largely consistent with the literature investigating sentence perception in normal and reading-impaired subjects (cf. Stollman et al., 1994). In one study, poor-reading children were impaired at discriminating phoneme contrasts embedded in sentences compressed at 50\% (Watson et al., 1990). In contrast, good readers were fairly accurate at this level of compression ( $>75 \%$ accuracy). In another study, learning-impaired children with auditory processing deficits were specifically deficient in speech recognition at $60 \%$ speech compression, while unimpaired children showed no effect of compression (McNutt and Chia-Yen Li, 1980). Although reading scores were not reported in the latter study, reading impairments affect $\sim 80 \%$ of learning-impaired individuals (Lerner, 1989; Lyon et al., 2003). Given the correspondence between current neurophysiologic results and previous behavioral findings, we propose that abnormal precision and magnitude measures of speech envelope representation in cortex underlie perceptual deficits for compressed speech.

Together, results from poor readers do not present a simple story. For one measure of speech envelope representation, poor readers' cortical responses were abnormal for all speech conditions. For the other two measures, poor readers were only differentiated from good readers in compressed speech. One possible explanation for these results is that these cortical measures represent a complex constellation of neural response features, all of which correlate with reading ability. These features include precision and magnitude measures that reflect perception of the speech envelope, as well as a general timing delay in the speech envelope mechanism that does not reflect perception, but may be related to other deficiencies in poor readers.

\section{Theories of reading impairment}

Despite decades of research, the neurobiological foundation of reading impairments remains elusive. While there is nearuniversal agreement that many poor readers suffer from a phonological deficit, whether or not phonological deficits are secondary to a more fundamental sensory deficit remains a source of debate (Rosen, 2003; Bishop, 2006; Goswami, 2006; White et al., 2006). While some argue for a causal relationship between sen- 
sory impairments and phonological difficulties (Goswami, 2002, 2006), others believe that phonological deficits are not causally related to sensory impairments (White et al., 2006). Therefore, the latter view requires that abnormal neurophysiological representation of the speech envelope and impaired perception for slow, nonspeech signals (Goswami, 2002; Witton et al., 2002; Richardson et al., 2004; Corriveau et al., 2007) share a common underlying biological factor with impaired reading and phonology, but are otherwise unrelated phenomena (White et al., 2006).

Here we show strong correlations between speech envelope processing and standardized measures of reading and phonological processing. If speech envelope representation is unrelated to reading ability, results would indicate that the putative "common biological factor" that accounts for these two phenomena varies in severity along a continuum for all readers, and that both envelope processing and phonology are affected similarly at points across this continuum, but are still unrelated. An arguably more convincing hypothesis is that temporal processing abilities vary along a continuum and affect phonological abilities and reading in a proportional manner, and representations measured in the current study reflect an important aspect of speech processing.

Those who view sensory deficits as an underlying factor to phonological impairments in poor readers (Stein and Walsh, 1997; Tallal et al., 1998) might view the current results as evidence that abnormal auditory representations of temporal elements of speech are more pervasive than previously thought in this population; they are not isolated to rapid components of speech. While abnormal representation of the speech envelope contradicts the temporal specificity of Tallal's hypothesis, this finding is consistent with the theory that impairments for temporal acoustic processing negatively impacts phoneme discrimination. A different set of conclusions can be drawn from a developmental theory of reading acquisition, which argues for sequential development of phonological abilities in young children, with word and syllablelevel skills preceding phoneme-level skills (Stanovich, 1992; Anthony et al., 2003; Ziegler and Goswami, 2005). From this perspective, phoneme-level deficits would represent a consequence of syllable deficits such as those shown here in poor readers.

A final consideration regarding theories of reading impairments is the heterogeneity of the reading-impaired population. Proponents of a non-sensory-based phonological deficit have argued that the failure for all reading-impaired individuals to exhibit auditory deficits indicates that these two factors cannot be related (White et al., 2006). Meanwhile, those who believe that auditory deficits contribute to reading impairments have not generally acknowledged that many poor readers do not suffer from auditory deficits. Perhaps it is the case that the deficits underlying reading impairments are as heterogeneous as the collection of sensory, cognitive, and mnemonic mechanisms required for normal reading acquisition. If so, auditory impairments such as those demonstrated here may only affect a subpopulation of reading-impaired individuals, and may serve to exacerbate this subgroup's phonological deficits (Bishop, 2006).

\section{Implications for remediation of reading impairments}

Much attention has been focused on remediating phonological disorders in poor readers. One approach has been to intensively train the discrimination of phoneme contrasts (i.e., rapid acoustic features), a method that has alternatively yielded impressive (Merzenich et al., 1996; Tallal et al., 1996; Moore et al., 2005) and disappointing (Cohen et al., 2005; Gillam et al., 2008; Given et al., 2008) results. Given that dyslexic children have shown greater sensitivity to allophones than controls (Serniclaes et al., 2004), it is plausible that improvements seen using this method may arise because a processing strength is being trained. Current results, in conjunction with previous findings (Stanovich, 1992; Anthony et al., 2003; Ziegler and Goswami, 2005), suggest that a key to phonological development lies with syllables. Therefore, additional benefit in phonological measures could potentially be achieved if training involves temporal cues involved in syllable-rate processing of speech.

\section{References}

Abrams DA, Nicol T, Zecker SG, Kraus N (2006) Auditorybrainstem timing predicts cerebral asymmetry for speech. J Neurosci 26:11131-11137.

Abrams DA, Nicol T, Zecker S, Kraus N (2008) Right-hemisphere auditory cortex is dominant for coding syllable patterns in speech. J Neurosci 28:3958-3965.

Ahissar E, Nagarajan S, Ahissar M, Protopapas A, Mahncke H, Merzenich MM (2001) Speech comprehension is correlated with temporal response patterns recorded from auditory cortex. Proc Natl Acad Sci U S A 98:13367-13372.

Anthony JL, Lonigan CJ, Driscoll K, Phillips BM, Burgess SR (2003) Phonological sensitivity: a quasi-parallel progression of word structure units and cognitive operations. Read Res Quart 38:470-487.

Banai K, Kraus N (2006) Neurobiology of (central) auditory processing disorder and language-based learning disability. In: Handbook of central auditory processing disorder: auditory neuroscience and diagnostics, Vol 1 (Chermak GD, Musiek FE, eds), pp 89-116. San Diego: Plural Publishing.

Banai K, Nicol T, Zecker SG, Kraus N (2005) Brainstem timing: implications for cortical processing and literacy. J Neurosci 25:9850-9857.

Banai K, Hornickel J, Skoe E, Nicol T, Zecker S, Kraus N (2009) Reading and subcortical auditory function. Cereb Cortex. Advance online publication. Retrieved May 11, 2009. doi:10.1093/cercor/bhp024.

Belin P, Zilbovicius M, Crozier S, Thivard L, Fontaine A, Masure MC, Samson Y (1998) Lateralization of speech and auditory temporal processing. J Cogn Neurosci 10:536-540.

Bellis TJ, Nicol T, Kraus N (2000) Aging affects hemispheric asymmetry in the neural representation of speech sounds. J Neurosci 20:791-797.

Bishop DV (2006) Dyslexia: what's the problem? Dev Sci 9:256-257; discussion 265-269.

Bradlow AR, Kraus N, Hayes E (2003) Speaking clearly for children with learning disabilities: sentence perception in noise. J Speech Lang Hear Res 46:80-97.

Brown L, Sherbenou R, Johnsen S (1997) Test of nonverbal intelligence: a language-free measure of cognitive ability. Austin, TX: Pro-Ed.

Cohen W, Hodson A, O’Hare A, Boyle J, Durrani T, McCartney E, Mattey M, Naftalin L, Watson J (2005) Effects of computer-based intervention through acoustically modified speech (Fast ForWord) in severe mixed receptive-expressive language impairment: outcomes from a randomized controlled trial. J Speech Lang Hear Res 48:715-729.

Corriveau K, Pasquini E, Goswami U (2007) Basic auditory processing skills and specific language impairment: a new look at an old hypothesis. J Speech Lang Hear Res 50:647-666.

Cunningham J, Nicol T, Zecker SG, Bradlow A, Kraus N (2001) Neurobiologic responses to speech in noise in children with learning problems: deficits and strategies for improvement. Clin Neurophysiol 112:758-767.

Drullman R, Festen JM, Plomp R (1994) Effect of temporal envelope smearing on speech reception. J Acoust Soc Am 95:1053-1064.

Gillam RB, Loeb DF, Hoffman LM, Bohman T, Champlin CA, Thibodeau L, Widen J, Brandel J, Friel-Patti S (2008) The efficacy of Fast ForWord Language intervention in school-age children with language impairment: a randomized controlled trial. J Speech Lang Hear Res 51:97-119.

Given BK, Wasserman JD, Chari SA, Beattie K, Eden GF (2008) A randomized, controlled study of computer-based intervention in middle school struggling readers. Brain Lang 106:83-97.

Goswami U (2002) Phonology, reading development and dyslexia: a crosslinguistic perspective. Ann Dyslexia 52:1-23.

Goswami U (2006) Sensorimotor impairments in dyslexia: getting the beat. Dev Sci 9:257-259; discussion 265-269.

Goswami U, Thomson J, Richardson U, Stainthorp R, Hughes D, Rosen S, Scott SK (2002) Amplitude envelope onsets and developmental dyslexia: a new hypothesis. Proc Natl Acad Sci U S A 99:10911-10916. 
Gustafson S, Samuelsson S (1999) Intelligence and dyslexia: implications for diagnosis and intervention. Scand J Psychol 40:127-134.

Jasper HH (1958) The ten-twenty electrode system of the international federation. Electroencephalogr Clin Neurophysiol 10:371-375.

Joanisse MF, Gati JS (2003) Overlapping neural regions for processing rapid temporal cues in speech and nonspeech signals. Neuroimage 19:64-79.

Kraus N, McGee TJ, Carrell TD, Zecker SG, Nicol TG, Koch DB (1996) Auditory neurophysiologic responses and discrimination deficits in children with learning problems. Science 273:971-973.

Lerner JW (1989) Educational interventions in learning disabilities. J Am Acad Child Adolesc Psychiatry 28:326-331.

Liégeois-Chauvel C, de Graaf JB, Laguitton V, Chauvel P (1999) Specialization of left auditory cortex for speech perception in man depends on temporal coding. Cereb Cortex 9:484-496.

Luo F, Wang Q, Kashani A, Yan J (2008) Corticofugal modulation of initial sound processing in the brain. J Neurosci 28:11615-11621.

Lyon GR, Shaywitz SE, Shaywitz BA (2003) A definition of dyslexia. Ann Dyslexia 53:1-14.

McNutt JC, Chia-Yen Li J (1980) Repetition of time-altered sentences by normal and learning disabled children. J Learn Disabil 13:30-34.

Merzenich MM, Jenkins WM, Johnston P, Schreiner C, Miller SL, Tallal P (1996) Temporal processing deficits of language-learning impaired children ameliorated by training. Science 271:77-81.

Moore DR, Rosenberg JF, Coleman JS (2005) Discrimination training of phonemic contrasts enhances phonological processing in mainstream school children. Brain Lang 94:72-85.

Nahum M, Nelken I, Ahissar M (2008) Low-level information and highlevel perception: the case of speech in noise. PLoS Biol 6:e126.

Phillips DP, Farmer ME (1990) Acquired word deafness, and the temporal grain of sound representation in the primary auditory cortex. Behav Brain Res 40:85-94.

Poeppel D (2003) The analysis of speech in different temporal integration windows: cerebral lateralization as 'asymmetric sampling in time'. Speech Commun 41:245-255.

Polley DB, Steinberg EE, Merzenich MM (2006) Perceptual learning directs auditory cortical map reorganization through top-down influences. J Neurosci 26:4970-4982.

Ramus F (2001) Dyslexia. Talk of two theories. Nature 412:393-395.

Richardson U, Thomson JM, Scott SK, Goswami U (2004) Auditory processing skills and phonological representation in dyslexic children. Dyslexia 10:215-233.

Rosen S (1992) Temporal information in speech: acoustic, auditory and linguistic aspects. Philos Trans R Soc Lond B Biol Sci 336:367-373.

Rosen S (2003) Auditory processing in dyslexia and specific language impairment: is there a deficit? What is its nature? Does it explain anything? J Phonetics 31:509-527.

Schlaggar BL, McCandliss BD (2007) Development of neural systems for reading. Annu Rev Neurosci 30:475-503.

Serniclaes W, Van Heghe S, Mousty P, Carré R, Sprenger-Charolles L (2004) Allophonic mode of speech perception in dyslexia. J Exp Child Psychol 87:336-361.

Song JH, Skoe E, Wong PC, Kraus N (2008) Plasticity in the adult human auditory brainstem following short-term linguistic training. J Cogn Neurosci 20:1892-1902.
Stanovich KE (1992) Speculations on the causes and consequences of individual differences in early acquisition. In: Reading acquisition (Gough PB, Ehri LE, Treiman R, eds), pp 307-342. Hillsdale, NJ: Erlbaum.

Stein J, Walsh V (1997) To see but not to read; the magnocellular theory of dyslexia. Trends Neurosci 20:147-152.

Stollman MH, Kapteyn TS, Sleeswijk BW (1994) Effect of time-scale modification of speech on the speech recognition threshold in noise for hearing-impaired and language-impaired children. Scand Audiol 23:39-46.

Tallal P (1980) Auditory temporal perception, phonics, and reading disabilities in children. Brain Lang 9:182-198.

Tallal P, Piercy M (1973) Defects of non-verbal auditory perception in children with developmental aphasia. Nature 241:468-469.

Tallal P, Miller S, Fitch RH (1993) Neurobiological basis of speech: a case for the preeminence of temporal processing. Ann N Y Acad Sci 682:27-47.

Tallal P, Miller SL, Bedi G, Byma G, Wang X, Nagarajan SS, Schreiner C, Jenkins WM, Merzenich MM (1996) Language comprehension in language-learning impaired children improved with acoustically modified speech. Science 271:81-84.

Tallal P, Merzenich MM, Miller S, Jenkins W (1998) Language learning impairments: integrating basic science, technology, and remediation. Exp Brain Res 123:210-219.

Tzounopoulos T, Kraus N (2009) Learning to encode timing: mechanisms of plasticity in the auditory brainstem. Neuron 62:463-469.

Vellutino FR, Scanlon DM, Lyon GR (2000) Differentiating between difficult-to-remediate and readily remediated poor readers: more evidence against the IQ-achievement discrepancy definition of reading disability. J Learn Disabil 33:223-238.

Watson M, Stewart M, Krause K, Rastatter M (1990) Identification of timecompressed sentential stimuli by good vs poor readers. Percept Mot Skills 71:107-114.

Wechsler D (1991) Manual for the Wechsler intelligence scale for children, Ed 3. San Antonio, TX: Psychological Corporation.

White S, Milne E, Rosen S, Hansen P, Swettenham J, Frith U, Ramus F (2006) The role of sensorimotor impairments in dyslexia: a multiple case study of dyslexic children. Dev Sci 9:237-255; discussion 265-269.

Wible B, Nicol T, Kraus N (2005) Correlation between brainstem and cortical auditory processes in normal and language-impaired children. Brain 128:417-423.

Wilkinson G (1993) Wide range achievement test-3 (WRAT-3). Wilmington, DE: Jastak Associates.

Witton C, Stein JF, Stoodley CJ, Rosner BS, Talcott JB (2002) Separate influences of acoustic AM and FM sensitivity on the phonological decoding skills of impaired and normal readers. J Cogn Neurosci 14:866-874.

Woodcock R, Johnson M (1977) Woodcock-Johnson psychoeducational battery: tests of cognitive ability. Allen, TX: DLM Teaching Resources.

Woodcock R, Johnson M (1989) Woodcock-Johnson psychoeducational battery revised: tests of cognitive ability. Allen, TX: DLM Teaching Resources.

Ziegler JC, Goswami U (2005) Reading acquisition, developmental dyslexia, and skilled reading across languages: a psycholinguistic grain size theory. Psychol Bull 131:3-29. 